\title{
Curcumin is Effective in Improving Folliculogenesis Profile (Primary and Secondary Folicles) and Oocytes Quality In Vitro in Ectopic Endometriosis Mice (Mus Musculus) Models
}

\author{
$\underline{\text { Aulia Firmawati }}{ }^{*}$, and Widjiati \\ ${ }^{1}$ Department of Veterinary Reproduction, Faculty of Veterinary Medicine, Brawijaya \\ University, Jl. MT Haryono Malang, Indonesia \\ ${ }^{2}$ Department of Embryology, Faculty of Veterinary Medicine, Airlangga University, $\mathrm{Jl}$ \\ Mulyorejo, Surabaya, Indonesia \\ *e-mail: auliafirmawatidrh@gmail.com
}

\begin{abstract}
Endometriosis is the presence of ectopic endometrial tissue, such as that outside the uterine cavity, which can cause chronic inflammatory reactions. Curcumin is one of traditional herbal medicines that is widely used. Some experiments have managed to find a mechanism to treat an ectopic endometriosis through the mechanism of suppression of several cytokines.This study was an experimental laboratory study, with four treatments and five replications, using female mice (Mus musculus) that had reached puberty. The study consisted of three phases: the first phase was the microscopic examination of in vitro oocytes quality, and the second was histopathological examination of folliculogenesis profile using Hematoxillin eosin staining. The results of this study showed significant difference between positive control group (P0) with treatment and negative control $(\mathrm{KN})$ groups in folliculogenesis profile ( $\mathrm{p}$ $<0.05)$. Examination of in vitro oocytes quality showed significant difference between positive control group with treatment group and negative control group ( $\mathrm{p}<0.05$ ). Curcumin has several roles in cytokines modulation in ectopic endometriosis mouse models. Curcumin may reduce the occurrence of apoptosis of granulosa cells so that it may directly improve oocyte quality and folliculogenesis profile (tersier follicel and de graf). In conclusion, curcumin effectively overcomes fertility problems through the mechanism of cytokines modulation that plays a role in some cases of endometriosis such as: TNF-alpha, NF-kB and COX-2. Curcumin may improve folliculogenesis profile and in vitro oocytes quality.
\end{abstract}

Keywords: Ectopic endometriosis, Curcumin, Folliculogenesis profile, Oocyte quality

\section{INTRODUCTION}

Fertility in female animals is closely related to fertilization rate. Both depend on the folliculogenesis process and the quality of the ovum. It needs physiological coordination in the ovary. Certain conditions may lead to a disruption in folliculogenesis, which may result in infertility. One major cause of infertility is the ovulation failure. Other circumstances that may be the most common cause of infertility is the formation of endometrial tissue on the outside of uterine cavity. This condition we commonly call with endometriosis (Herliana et al., 2008). Endometriosis is a health problem that affects at least $10 \%$ in women of childbearing age, $20-50 \%$ in infertile women and may also occur in baboons (Papio anubis) and also the long-tailed macaque (Macaca fascicularis) with a prevalence rate of $85 \%$. Endomteriosis in baboons (Papio anubis) and the long-tailed macaque (Macaca fasiculata) occurrs because the two animals have common features with female humans in terms of the 
shape and pelvic anatomic shape and uterine type, reproductive and immunological physiological characteristics (Grummer, 2006). In endometriosis model extracellular matrix binding, which constitue bonds cumulus oophorus, is thickening (Herliana et al., 2008), and granulosa cell may become apoptotic, which is also suspected as the cause of the decline in oocyte quality. This adversely affects the fertility in the animal models. In addition, in the case of endometriosis follicular period is extending, follicular growth is low and the size of the dominant follicle in endometriotic women is decreasing (Gupta, 2008).

Rats (Rattus norwegicus) and mice (Mus musculus) model of endometriosis show low fertility and the presence of nodules on pelvic region. Permana (2007) found that mice (Mus musculus) had decreased lymphocyte function, by mechanisms that are allegedly due to the suppression of the proliferation and differentiation of lymphocytes, also a feature of angiogenesis in uterine area, ovaries and peritoneal front wall. Today, herbal remedies increasingly popular are increasingly popular, used also for alternative therapies in the field of gynecological diseases, such as endometriosis and ovarian carcinoma. This is because herbal medicines do not have any significant side effects compared to synthetic drugs in the market during this time. One of the traditional herbal medicines which have been used to treat endometriosis is curcumin, an active ingredient extracted from Curcuma longa. Curcumin is a natural yellow pigment of turmeric. Its content is approximately $3-4 \%$ of turmeric. Curcumin has also been shown to function as anti-inflammatory, anti-oxidant and anti-cancer. Some experimental test has found the mechanism by which curcumin treats endometriosis, which is through the suppression of some of proinflammatory cytokines, such as TNF-alpha, barriers COX-2 and NF-kappabeta (Wiesser et al., 2007). In this study, we used experimental animals SCID (Severe Combined Immunodeficient) mice (Mus musculus) as an endometriotic model. Awwad et al., (1999) had successfully performed the implantation of endometriosis in mice (Mus musculus) with a success rate of 96.5\%. We, therefore, investigated the effectiveness of curcumin in endometriosis treatment. Until then, studies on the effectiveness of curcumin in ectopic endometriosis mouse (Mus musculus) model on fertility have never been done.

\section{METHODS}

The study was conducted at in vitro fertilization laboratory and histopathological examination was conducted at the Laboratory of Anatomic Pathology, Faculty of Veterinary Medicine, Universitas Airlangga, Surabaya. The study was performed for three months. Experimental animals were 20 virgin female mice aged 3 months with an average weight of 20-30 grams. Vasectomized male mice were as many as 20 mice and adult male mice were 4. Materials used in this study were as follows: 0.9\% physiological Sodium Chloride $(\mathrm{NaCl})$, Diionize Water (DW), Phosphate Buffer Saline (PBS), MEM, endometrial tissue of cysts model at Dr. Soetomo Hospital, Surabaya, penicillin, streptomycin, estradiol, curcumin, cyclosporin A and mineral oil, 70\% alcohol, sterile paper tisuue, and distilled water. Equipments used in this study were as follows: Pasteur pipette, Eppendroff, disposable petri dish sized $30 \times 80 \mathrm{~mm}$, glass petri dish, $1 \mathrm{ml}$ and $5 \mathrm{ml}$ disposable syringes, laminar flow, inverted microscopes, centrifuges, Bunsen burners, analytical balance, 5\% CO2 incubator with a temperature of 38.5 degrees $\mathrm{C}$ and a digital camera.

The making of endometriotic mouse models. First, the immune system of the mice was suppressed using cyclosporine A. After reconstitution with water for injection, cyclosporine A was injected on day 1 intramuscularly in femoral biceps muscles at a dose of $1.8 \mathrm{mg} / \mathrm{mouse}$. Then, estrogen was injected intramuscularly in femoral biceps muscles on day 1 and 5 after the injection of female treatment endometrium. Thereafter, implantation of endometrial tissue was done on day-1 by injecting endometrial isolate intraperitoneally as 
much as $0.1 \mathrm{cc}$, equivalent to 0.6 grams of wet endometrial tissue (Awwad et al., 1999). Folliculogenesis profile. Examination was done by taking the right and left ovary. Furthermore, both ovaries were made as histological preparations and primary and secondary follicle counts in the ovaries were observed under a light microscope with a magnification of 400 times. Each ovary was made into three preparations, the anterior, medial and a third posterior part. The result was the sum of total count observed in both ovaries. Oocyte quality. Examination was done by collecting oocytes from fertilization pouch, then oocytes that remained with Cumulus Oophorus Complex (COC) was photographed using digital camera Canon Ixus 95 I inverted microscope magnification 100x and camera magnification 3,0x. Thereafter, COC circumference width was measured using the software Miotic Image II Plus.

\section{RESULTS AND DISCUSSION}

\section{Folliculogenetic profile}

Determination of folliculogenesis development in the ovary of endometriotic mouse (Mus musculus) was done with a series of microscopic observation to count the number of primary, secondary, tertiary and de Graaf's follicles. Observation and calculation of follicle count was based on the desired characteristics possessed by each follicle (Table 1).

Table 1. Folliculogenesis profile.

\begin{tabular}{|c|c|c|}
\hline \multirow{2}{*}{ Treatment } & \multicolumn{2}{|c|}{ Folliculogenesis profile } \\
\cline { 2 - 3 } & Primary follicles & Secondary follicles \\
\hline KN & $17.00^{\mathrm{a}} \pm 5.83$ & $21.83^{\mathrm{ab}} \pm 4.44$ \\
\hline P0 & $19.16^{\mathrm{a}} \pm 4.21$ & $30.83^{\mathrm{a}} \pm 8.01$ \\
\hline P1 & $18.16^{\mathrm{a}} \pm 6.36$ & $27.83^{\mathrm{a}} \pm 2.63$ \\
\hline P2 & $16.33^{\mathrm{a}} \pm 6.50$ & $24.50^{\mathrm{ab}} \pm 7.20$ \\
\hline
\end{tabular}

of a highly significant difference $(\mathrm{p}<0.05)$ with LSD

The results showed that the group of ectopic endometriosis mice receiving placebo (P0) and the group receiving curcumin of $24 \mathrm{mg} / \mathrm{kg}$ bw/day (P1) had the highest mean primary and secondary follicle count among the negative control group and other treatment groups. It may result from extended periods of follicular period due to granulosa cell apoptosis. This extension lead to follicular growth disorder characterized by oocyte follicle growth disorders, oocytes proliferation and differentiation. It indirectly results in decreased FSH and GDF-9 levels. This condition leads to resisted expressing mechanisms of FSH receptor, activin and GDF-9 produced by the granulosa cells, that are thought to play a role in stimulating FSH receptor expression through autocrine/paracrine mechanism.

Decreased FSH and GDF-9 levels on granulosa cell due excessive apoptosis in this granulosa cells have caused disruption of enzymatic activity that is useful for catalyzing androgens aromatization to produce estrogen, so it causes inhibited steroidogenesis process and decreased estrogen sensitivity to provide feedback to the pituitary anterior so that GnRH may produce LH. As a result, this will affect oocyte maturation process resulting, in extended follicular periods that results in folliculogenesis disturbance in the positive control group (P0). In treatment group 1 (P1) mean primary and secondary follicles counts were high. This may be because the administration of curcumin doses in this group was less effective, so that the process within the inhibition of some cytokines such as TNF-alpha, COX-2 and NF-kB less than optimal in improving the condition of ectopic endometriosis. The primary follicle profile can be seen in Figure 1. 

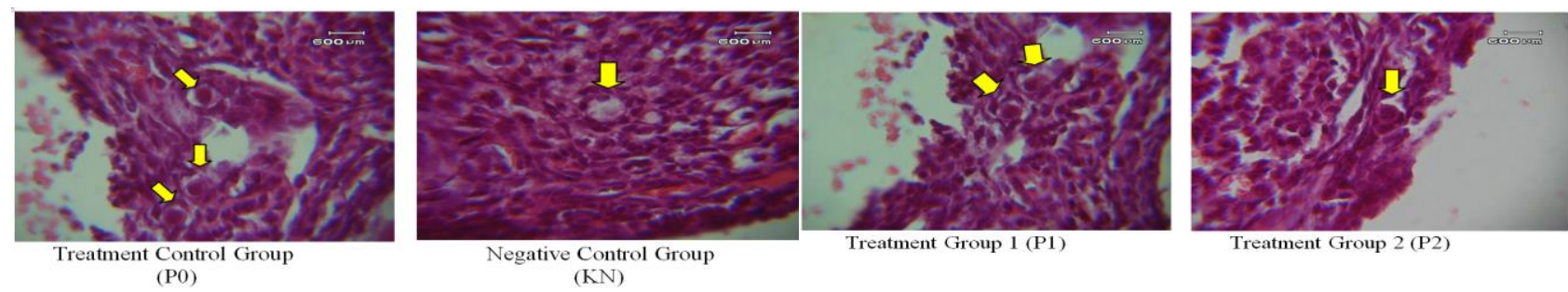

Figure 1. Folliculogenesis profile. Forms of primary follicles in each of the control and treatment groups. Observation was made at 400x microscope magnif tion. Sign ( ) indicates the shape of primary follicles.

Curcumin is one of the herbal remedies that can suppress NF-kB and cytokines inflamatory pathway and also target genes of NF-kB cytokines in endometriotic models. Wieser et al., (2007) demonstrated curcumin effects on endometriotic stromal cells. Curcumin inhibits the induction of pro-inflammatory cytokines, angiogenic cytokines and macrophage migration inhibitory factor by NF-kB in in vitro model. Several recent studies also found the modulatory effect of curcumin on several important molecular targets (TNF, IL-1, IL-6) and enzyme (COX-2) (Wieser et al., 2007). In addition, curcumin can also lower anti-apoptotic genes expression, antioxidants and anti-angiogenesis effects (Sharma et al., 2005; Joe et al., 2008). Inhibition of excessive TNF-alpha secretion and pro-inflammatory cytokines through suppression of NF-kB and NF-kB target gene cytokines pathway by curcumin in ectopic endometriosis mouse model of through modulation mechanism of several pro-inflammatory molecules targets in ectopic endometriosis mouse model, particularly molecule targets TNF-alpha, enzyme cyclooxygenase-2 (COX-2) and xanthine oxidase, which can reduce the occurrence of apoptosis of granulosa cells, which may increase FSH level along GDF-9, which may enhance follicular development. The secondary follicles profile can be seen in Figure 2.
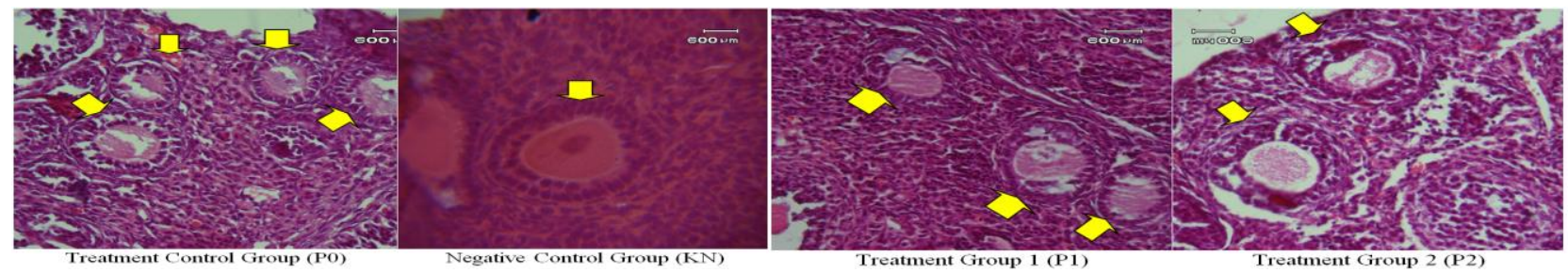

Figure 2. Folliculogenesis profile of secondary follicles in each control and treatment groups. Observations made at 400x micro Dpe magnification. Sign ( ) indicates the shape of secondary follicles.

Increased FSH level on granulosa cells may enhance enzymatic activity useful for catalyzing androgens aromatization or the like to produce estrogen. These activities are suggested to be organized by an increase in adenylate cyclase and androgen action. Estrogen (estradiol), which is synthesized by the dominant follicle, also plays a role in increased FSH follicular cells action to improve LH response. In addition to producing estrogen, FSH also plays a role in egg maturation, especially on the stages of follicular change. In addition, GDF9 level in granulosa cells may increase so that GDF-9 is capable of running proliferation and differentiation of granulosa cells so well that the proliferation of theca cells will not be disturbed. Interstitial theca cells have cell receptors for luteinizing hormone and insulin, in response to LH and insulin stimulation. The cells may produce high levels of androgens (Anwar, 2005). 


\section{Oocyte quality}

Determining the quality of oocytes was done by observing the oocyte microscopically to observe cumulus ooporus circumference (Table 2). Based on Analysis of Variant (ANOVA) followed by LSD test it has been found that the group endometriosic ectopic mice model receiving curcumin at $36 \mathrm{mg} / \mathrm{kg} /$ day $(\mathrm{P} 2)$ were highly significantly $(\mathrm{p}<0.05)$ different from ectopic endometriosis mice model receiving curcumin at $24 \mathrm{mg} / \mathrm{kg}$ bw/day (P1) and mice without treatment $(\mathrm{KN})$.

Table 2. Oocytes quality

\begin{tabular}{|c|c|c|}
\hline Treatment & $\begin{array}{c}\text { Oocytes count } \\
\text { from IVF }\end{array}$ & Oocye quality observation \\
\hline KN & 134 & $2,98^{\mathrm{b}} \pm 0,239$ \\
\hline P0 & 0 & $0^{\mathrm{c}} \pm 0$ \\
\hline P1 & 94 & $3,07^{\mathrm{b}} \pm 0,125$ \\
\hline P2 & 124 & $3,60^{\mathrm{a}} \pm 0,256$ \\
\hline
\end{tabular}

Notes: ${ }^{a, b, c}$ : different superscript in the same column indicate the presence of a highly significant difference ( $\mathrm{p}<0.05)$ with Tukey's HSD test.

It shows that curcumin administration to ectopic endometriosis mouse models can improve oocytes quality (cumulus oophorus complex circumference) by inhibiting apoptosis of granulosa cells through modulation mechanism of several targets of pro-inflammatory molecules ectopic endometriosis mouse models, especially TNF-alpha, enzyme cyclooxygenase-2 (COX-2) and xanthine oxidase so as to increase GDF-9 level and decrease hyaluronant level in ectopic endometriosis mouse models. Inhibition mechanism of molecule target in ectopic endometriosis mouse models with curcumin is expected to improve the proliferation and differentiation of granulosa cells, that can also improve cumulus expansion which may directly improve oocyte quality by increasing Cumulus Oophorus Complex (COC) circumference.

\section{REFERENCES}

1. Anwar, R. 2005. Morfologi dan Fungsi Ovarium. Subbagian Fertilitas dan Endokrinologi Reproduksi. Fakultas Kedokteran Unniversitas Padjajaran. Bandung.

2. Awwad J.T., RA Sayegh, XJ Tao. The SCID mouse : an experimental model for endometriosis. Human Reproduction. 1999 ; 14 (12) : 3107 - 11

3. Grummer R .2006. Animal models in endometriosis research; human reproduction update vol 12, no 5 pp.641-649.

4. Gupta,S. 2008. Pathophisiology, infertility of endometriosis. http://endometriosis.edu.html. 